\title{
Pour une sémantique des valeurs archétypales : le cas des mots croisés N17 de Georges Perec
}

\author{
Chantova, Yasena \\ Sens, Texte, Informatique, Histoire (STIH), Université Paris-Sorbonne, 1 rue Victor Cousin, 75005 Paris - France \\ iasena chantova@yahoo.com
}

Les grilles des mots croisés, parce qu'elles rappellent de prime abord celles d'un échiquier, évoquent immédiatement, chez un linguiste, le célèbre exemple de Saussure (1955[1916]) et sa notion de valeur. D'une part, toute valeur résulte des rapports systémiques avec d'autres valeurs. Elle est d'autre part une association entre son signifiant et son signifié. Dans la perspective de la notion de valeur, l'approche suivante des mots croisés devient possible: considérer les réponses d'une grille comme valeurs saussuriennes pour aborder les rapports associatifs entre elles et pour envisager les associations, qui s'établissent entre ces réponses et leurs définitions.

Les rares ouvrages, qui s'engagent dans l'analyse de ce genre de texte, traitent du rapport associatif entre la définition et la réponse : soit l'approche se fait en termes de permutation et de substitution afin d'établir une isotopie possible entre la définition et la dénomination (Greimas, 1970:285-308), soit une analyse relationnelle est proposée (Wittwer, 2004).

Un bref article (Attali, 2008:67-71) traite de l'humour dans certaines définitions des mots croisés de G. Perec par rapport à leurs réponses. Et un autre article (Örsi, 1996:93-99) envisage quelques procédés lexicologiques (polysémie, synonymie, homonymie, métaphore) d'élaboration des définitions en vue des réponses dans des mots croisés français.

À part qu'ils fournissent une présentation générale du phénomène des mots croisés (leur origine, leur utilisation sociale, l'architecture des grilles, les principales variantes de mots croisés et d'autres formes de croisements de mots), La Ferté \& Capelovici (1975) abordent aussi les types de définitions sans quitter cependant le rapport de celles-ci avec les réponses. Toutefois, il est possible d'entrevoir un certain rapport entre les réponses elles-mêmes dans les mots croisés à thème (La Ferté \& Capelovici, 1975:44-47) : par ex., les réponses d'une même grille relèvent du vocabulaire littéraire ou historique.

À son tour, Jeandillou (1995:75-96) met ce rapport d'équivalence dans la perspective du dialogisme et cherche à relever des modèles d'engendrement des définitions dans les mots croises de G. Perec. Ce sont, d'une part, les phénomènes de polysémie et d'homonymie, qui encodent les définitions : celles-ci deviennent alors plurivoques. C'est, d'autre part, l'autonymie, qui exploite la réflexivité du langage : les définitions, qui sont engendrées par la lecture réversible et anagrammatique, par de différentes troncations (apocopes, aphérèses, syncopes) et par les procédés de synecdoque, ont alors un fonctionnement métalinguistique. Finalement, les mots croises de G. Perec, parce qu'ils renvoient à d'autres textes, sont à considérer métatextuels. Les possibilités de pareils renvois quittent alors le simple rapport entre la définition et la réponse et empiètent sur les rapports associatifs entre plusieurs valeurs.

Il est possible ainsi d'aborder les associations entres les réponses d'une même grille au moins. Ces mots se croisent-ils uniquement au niveau de leurs signifiants ? Alors, il est possible d'élaborer un Dictionnaire des mots croisés, mots fléchés \& autres jeux de lettres (Le Robert, 2006) à la base des signifiants : la recherche de la réponse s'y fait à la suite de sa longueur (le nombre des lettres) et les positions des lettres. Une telle méthode adoptée exclut la possibilité d'une approche sémantique de ces mots. Cependant, ce dictionnaire englobe l'ensemble de la nomenclature du Nouveau Petit Robert où les signifiés de ces mots sont mis en jeu.

Les mots croisés, s'associent-ils au niveau de leurs signifiés? Alors, il est possible d'organiser un Dictionnaire des mots croisés et fléchés (Larousse, 2011) à la base des associations hiérarchiques des signifiés : les réponses possibles se trouvent regroupées sous des mots-clefs, auxquels le cruciverbiste accède par des renvois. Pour faciliter encore la recherche, des définitions simples séparent les majeures articulations du sens dans le signifié d'un mot-clef. Le nombre des lettres n'y est pas complètement exclu, 
mais les signifiants ne priment plus. Un tel dictionnaire, du point de vue de la notion de valeur, assure l'association des réponses sous un mot-clef, et par les renvois, l'association des réponses rangées sous plusieurs mots-clefs.

Afin d'y trouver la solution I horizontalement des mots croisés N17 de G. Perec, il suffit de partir de la définition ORDONNANCE, qui renvoie le cruciverbiste à cinq mots-clefs. La réponse CLASSEMENT (nom, 10 lettres) se trouve rangée sous la deuxième définition simple Disposition d'éléments du mot-clef ORDRE. La réponse n'est pas toutefois si facilement accessible. Et même, il est impossible de trouver la solution MERE (9 verticalement, N17) à l'aide des renvois FAIT VINAIGRE et VINAIGRE : le parcours associatif proposé par le dictionnaire diffère du celui de l'auteur-cruciverbiste et s'avère ainsi insuffisant.

L'aspect social, et même psychique, du phénomène des mots croisés, est souvent pris en considération. C'est ainsi qu'il est question d'une communication cruciverbiste avec des processus d'encodage et de décodage chez Greimas (1970:285-287). Selon Wittwer (2004:7-8), le côté psychosocial résulte, à la fois, des opérations mentales de compréhension et de décodage et des pratiques sociales des mots croisés. La Ferté \& Capelovici (1975:98-117) consacre ainsi tout le chapitre VI sur leur usage social (publicités, jeux télévisés, concours). Et la perspective du dialogisme, qui encadre l'approche de Jeandillou (1995:75-96), évoque aussi l'aspect social du phénomène. Quant à cet aspect des mots croisés dans les dictionnaires, il se voit confirmé dans la mesure où un dictionnaire relève d'un usage social.

Pour affiner le parcours associatif entre la définition et la réponse et pour aborder les rapports associatifs entre les réponses elles-mêmes, une approche sémantique en termes de valeurs des mots croisés N17 de Georges Perec (2012 [1999]) s'impose.

Considérée comme négative, la valeur saussurienne exclut toute détermination positive dans une perspective systémique et différentielle (Saussure, 1955 [1916]:155-165). Pourtant, à la suite du fragment 29j (Saussure, 2002:87-88), il est possible de considérer la valeur comme un «phénomène double» opérant en deux temps. Ainsi, sa négativité intervient toujours au commencement pour générer ensuite un fait de postélaboration:
«Le phénomène d'intégration [...] est le phénomène double qui résume toute la vie active du langage et par lequel
1. les signes existants évoquent MECANIQUEMENT, par le simple fait de leur présence et de l'état toujours accidentel de leurs DIFFERENCES à chaque moment de la langue, un nombre égal non pas de concepts, mais de valeurs opposées pour notre esprit [...]; cette opposition de valeurs qui est un fait PUREMENT NEGATIF se transforme en fait positif, parce que chaque signe en évoquant une antithèse avec l'ensemble des autres signes comparables à une époque quelconque, en commençant par les catégories générales et en finissant par les particulières, se trouve être délimité, malgré nous, dans sa valeur propre. Ainsi, dans une langue composé au total de deux signes, $b a$ et la, la totalité des perceptions confuses de l'esprit viendra NECESSAIREMENT se ranger ou sous $b a$ ou sous $l a$. [...]; à ce moment la somme de sa connaissance positive sera représentée par le caractère commun qu'il se trouve avoir attribué aux choses $b a$ et le caractère commun qu'il se trouve avoir attribué aux choses $l a$; ce caractère est positif, mais il n'a jamais cherché en réalité que le caractère négatif qui pût permettre de décider entre $b a$ et $l a ;[\ldots]$
Dans chaque signe existant vient donc S'INTEGRER, se postélaborer une valeur déterminée $[\ldots]$; et comme le nombre et l'aspect réciproque et relatif de ces signes changent de moment en moment d'une manière infinie, le résultat de cette activité, pour chaque signe, et pour l'ensemble, change aussi [...] dans une mesure non calculable. » (souligné - n.) (Saussure, 2002:87-88)

C'est notamment la notion de postélaboration qui permet l'approche sémantique. Consistant à intégrer dans les valeurs elles-mêmes leurs rapports différentiels et systémiques, cette postélaboration fait preuve d'une nature relative. Une sémantique des valeurs doit par conséquent prendre en considération cet aspect double de la valeur. 
Les notions ou «vérités fondamentales» étant interdépendantes chez Saussure (2002:17), cette postélaboration se voit aussi liée à des notions mieux connues, comme celle d'association (Saussure, 1955 [1916]:170-180). Se forment ainsi des groupes associatifs où toutes les valeurs partagent, au sein d'un même groupe, une valeur commune qui rappelle bien le caractère commun attribué aux « choses la » dans l'exemple de Saussure. Étant à la base des entités abstraites (Saussure, 1955 [1916]:189-192), ces associations de valeurs concrètes permettent la catégorisation grammaticale de même que la définition des catégories $b a$ et la. D'autre part, en établissant le rapport signifié - signifiant, l'association rend possible l'unité du signe linguistique, qui se voit défini en tant que terme positif (Saussure, 1955 [1916]:166-169).

Résultat de l'état du système langagier à un moment et dans un espace donnés, chaque postélaboration détermine l'identité d'une valeur en conservant ainsi le dynamisme du système et l'incalculabilité des valeurs. Ce qui justifie d'ailleurs que le Cours considère les identités comme coïncidant avec les valeurs, bien que ces identités soient les contreparties des différences (Saussure, 1955 [1916]:150-154).

Ne se préoccupant que du caractère distinctif des valeurs, Saussure n'apporte pas d'autres précisions sur la valeur postélaborée. Dans le présent travail, nous irons chercher ces précisions dans les notions d'identités générique, spécifique et numérique, attribuées à la suite de la division des quatre prédicables que sont la définition, le genre, le propre et l'accident d'Aristote (Top.). Toute division se faisant par les différences et toute différence étant de nature qualitative, ces différences opèrent une première fois de manière distinctive lors de la division. Elles participent ensuite à la détermination positive de chacune des prédicables en leur attribuant des identités, de sorte qu'une hiérarchie entre les prédicables, mais aussi entre les identités, est instaurée. Étant ainsi une opération double, la division rapportée dans le domaine des valeurs permettra que celles-ci se précisent comme génériques, spécifiques et numériques. Ces valeurs vont partager alors une valeur commune, pourvu qu'elles soient issues d'une même division.

Toute division établit de plus des rapports de type partie-tout entre les prédicables, et ainsi entre les identités, de sorte que chaque prédicable et chaque identité participent à titre constitutif dans la détermination de sa supérieure.

Dans le domaine linguistique, la nature qualitative et marquée d'une partie se voit justifiée par Jakobson. D'abord, par sa notion de marque (Jakobson, 1963:176-196) : c'est la présence d'une qualité quelconque qui permet de désigner un terme comme marqué, tandis que l'absence de cette qualité détermine le terme non-marqué. La marque est de plus une notion différentielle car elle distingue les deux termes dans leur opposition qualitative. D'autre part, la nature du rapport entre le tout et ses parties (Jakobson, 1971:280288) peut aussi être qualitative car c'est la partie qui détermine, c'est-à-dire marque, la nature du tout : un tel rapport s'installe ainsi entre le tout du signe et ses parties que sont le signifié et le signifiant. Ces considérations jakobsoniennes appliquées aux valeurs saussuriennes montrent que celles-ci sont des parties qualitatives et marquées.

Des associations hiérarchiques d'identités sont d'autre part mises en jeu lors de l'organisation catégorielle bidimensionnelle (Kleiber, 1990 et 1994). La dimension verticale étant régie par une relation d'implication, les occurrences à identité spécifique se définissent en tant que catégories hyponymiques par rapport à celles d'identité générique et en tant que catégories hyperonymiques par rapport à celles d'identité numérique. De là résultent, d'une part, la possibilité pour des identités de participer à l'expression métalinguistique, comme le font les entités abstraites saussuriennes et, d'autre part, le caractère dynamique de la notion de catégorie qui rappelle bien les changements non calculables des valeurs postélaborées. C'est la relation d'incompatibilité qui régit la dimension horizontale discursive en assurant une diversité des occurrences.

Un tel dynamisme catégoriel implique des précisions non seulement sur les types d'identités mais aussi sur les types de différences. L'établissement de la contradiction $(\mathrm{A}-\overline{\mathrm{A}} /$ non- $\mathrm{A}-$ non- $\overline{\mathrm{A}})$, de l'implication $(\overline{\mathrm{A}} \supset$ non-A / non- $\overline{\mathrm{A}} \supset \mathrm{A})$ et de la complémentarité $\left(\overline{\mathrm{A}}^{*} \supset \text { non-A*/ non- } \overline{\mathrm{A}}^{*} \supset \mathrm{A}^{*}\right)^{1}$ et finalement de la contrariété (A - non-A) (Greimas \& Courtés, 1979:29-33) permet de pareilles précisions. De plus, le carré sémiotique, qui respecte le principe saussurien des différences, n'est pas contradictoire avec la notion de valeur. 
Une telle approche des valeurs saussuriennes, qui reste de plus dans la perspective systémique, leur permettra de se postélaborer en catégories dont les dimensions verticales s'avèreront généralisatrices, tandis que les dimensions horizontales se chargeront de la différenciation et de la diversité discursive.

Finalement, il ne faut pas oublier la nature psychique et collective des valeurs linguistiques, celles-ci existant dans la conscience des sujets parlants (Saussure, 2002). Telle est d'autre part la nature des archétypes selon Jung (1971), qui ont une existence dans l'inconscient collectif. C'est cette nature psychique et collective qui permet une compatibilité des deux notions de valeur et d'archétype.

Les textes jungiens étant un discours, et même un discours scientifique, les archétypes, par exemple celui de la Mère, sont à considérer comme des occurrences discursives, autrement dit des valeurs saussuriennes.

Il est d'autre part possible de considérer ces archétypes comme des définitions aristotéliciennes (Top., I,5.) bien qu'en principe un seul mot ne constitue pas une définition : est finalement appelé définitionnel ce qui résulte de la méthode des définitions, une définition étant supposée porter sur les identités et les différences. La Mère, comme définition, doit alors faire preuve des différences qui assurent sa division et que sont ses qualités archétypales, ainsi que des identités qu'elle permet d'attribuer à ses manifestations. Si la Mère est posée en définition, c'est en vue de sa fonction. De plus, la notion de mot implique toujours des précisions.

La Mère se différencie des autres archétypes par ses manifestations (Jung, 1971:96-101). Parmi celles-ci, MER, MERE, CIEL, TERRES, UNIVERSITE figurent parmi les réponses des mots-croises $\mathrm{N} 6,17^{2}, 18$, 28 et 93 du recueil de Georges Perec (2012 [1999]). Il convient alors d'attribuer à ces cinq une fonction distinctive, en tant que manifestations de la Mère. D'autre part, puisqu'elles sont des réponses, autrement dit des occurrences au niveau du discours cruciverbiste, ces cinq sont aussi des valeurs saussuriennes. Par conséquent, elles se définissent en tant que valeurs différentielles. De plus, toute définition renfermant en soi les seuls genres et différences qui sont prédiqués à titre essentiel (Top., VII,3.), ces cinq valeurs acquièrent des identités génériques et sont ainsi des parties du tout de la Mère.

Toute division appliquée aux notions linguistiques ayant lieu au niveau sémantique (Kleiber, 1994), autrement dit au niveau du signifié saussurien, l'on peut poursuivre la division de la réponse, et par conséquent de la valeur générique MERE, grâce à sa définition cruciverbiste : FAIT VINAIGRE. Dans le domaine des mots-croisés, ce sont les définitions cruciverbistes qui fonctionnent comme des signifiés. Cependant, au-delà de ce signifié, un recours aux définitions proposées par le dictionnaire s'impose. Afin d'éviter toute sortie sur des contenus encyclopédiques, nous avons choisi les définitions proposées par le Petit Robert, dictionnaire alphabétique et analogique de la langue française (2013). Ces définitions constituent à leur tour des signifiés, qui renferment les formules définitionnelles correspondantes, les étymologies, les remarques métalinguistiques, les renvois analogiques, les contraires, les homonymes. L'hyperonymie et l'hyponymie sont mises en jeu, et assurent une arborescence sémantique. Grâce à cette arborescence l'expression familière FAIT VINAIGRE se définit par SE DEPECHER qui renvoie à SE PRESSER et ainsi de suite pour aboutir finalement à :

MERE - FAIT VINAIGRE - SE DEPECHER - $\Rightarrow$ SE PRESSER - PRESSER - TOURMENTER - SE TOURMENTER ANGOISSE

- VINAIGRE - MERE DE VINAIGRE - GELATINEUSE - de GELATINE - SUBSTANCE - TENIR

-1 DESSOUS - de

- ALCOOLIQUE - de ALCOOL

- FERMENTATION - TRANSFORMATION 
Ainsi, à partir de la valeur générique MERE se différencient deux associations initiales qui commencent par les valeurs spécifiques FAIT VINAIGRE et VINAIGRE. La seconde se différencie à son tour dans les valeurs GELATINEUSE, ALCOOLIQUE et FERMENTATION. Enfin, l'association issue de GELATINEUSE se divise entre les valeurs TENIR et 1. DESSOUS. Les valeurs ANGOISSE, TENIR, SOUS, ALCOOL et TRANSFORMATION étant les dernières issues de la division de la valeur générique MERE, elles sont toutes numériques et semblent bien avoir assuré la division distinctive car, si leur présence dans les signifiés de leurs hyperonymes et les valeurs spécifiques SE TOURMENTER, SUBSTANCE, 1. DESSOUS, ALCOOLIQUE et FERMENTATION sont plutôt «visibles », il suffit de remplacer dans GELATINE la valeur SUBSTANCE par le signifié de celle-ci contenant déjà TENIR, pour se rendre compte que la valeur GELATINE ainsi élaborée retient elle aussi cette valeur numérique.

Il est d'autre part possible de distinguer des niveaux de différenciation : toute intervention d'une nouvelle valeur numérique entraîne un changement de niveau. Ainsi, un niveau de différenciation A., B., ... débute avec FAIT VINAIGRE (A.) et VINAIGRE (B.), puisqu'elles sont définies par de valeurs numériques

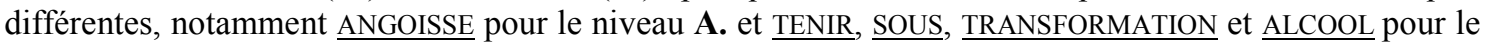
niveau B. Celui-ci se différencie encore en des sous-niveaux 1., 2. et 3. qui commencent dans GELATINEUSE, ALCOOLIQUE et FERMENTATION. La différenciation va encore plus loin dans le cas de GELATINEUSE pour constituer un nouveau niveau hyponymique a. et b.. Ainsi, l'articulation du sens assurée par les valeurs archétypales se constitue aussi de façon hiérarchique.

De pareilles postélaborations se font aussi pour le reste des valeurs initialement différenciées, de sorte que le signifié postélaboré de la générique MERE se voit marqué par la présence de ces numériques. Celles-ci s'avèrent alors être des parties marquées de chaque signifié où elles sont présentes. Elles sont ainsi les valeurs partagées par tous les membres de l'association catégorielle. En participant d'autre part aux postélaborations des termes hyperonymiques, elles marquent ainsi le caractère abstrait et le fonctionnement métalinguistique de ceux-ci. Quant à leur nature qualitative assurant à la fois la différenciation et la postélaboration, il suffit de remarquer qu'angoisse, soutient et transformation magique sont des qualités de l'archétype de la Mère (Jung, 1971:97), et que leurs signifiés fournissent des

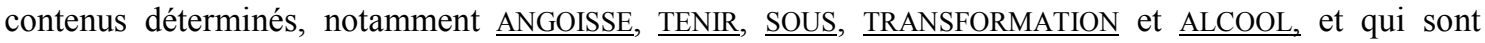
nécessaires aux postélaborations :

angoisse

ANGOISSE

soutient

SOUTENIR - de SOUS

- de $\underline{\text { TENIR }}$

transformation magique

TRANSFORMATION

MAGIQUE - $\Rightarrow$ ELIXIR - ALCOOLAT - de ALCOOL

Il en résulte que la différence qualitative assure l'articulation du sens.

Ces qualités et ces manifestations se différencient lors de leur opposition, de même que les deux signes la et $b a$. Cependant, si les manifestations archétypales ont leurs occurrences-réponses dans le discours cruciverbiste, les qualités se présentent implicitement dans ce discours : c'est ainsi que les associations formées au sein des signifiés des qualités sont des continuations des associations constituées dans les signifiés des occurrences.

Si la valeur numérique d'ANGOISSE, TENIR, SOUS, TRANSFORMATION, et ALCOOL ainsi que la valeur générique de MERE semblent justifiées, l'attribution d'une valeur spécifique au reste des membres de cette catégorie paraît plutôt discutable. A moins que l'on ne se place dans la perspective de leurs rapports hyponymiques et hyperonymiques. Si la valeur SUBSTANCE s'avère spécifique par rapport à son 
hyponyme TENIR, elle devient à son tour, parce qu'elle le contient dans son signifié, numérique et hyponymique par rapport à GELATINE, tout en contribuant ainsi au dynamisme de la catégorie. Et il en est de même pour le reste des valeurs.

S'il apparaît d'autre part que l'ordre et le nombre des valeurs sont bien fixés, à la différence de ceux des groupes associatifs chez Saussure (1955 [1916]:170-180), cela est plutôt dû au fait que les signifiés fournis par le dictionnaire, renfermant des réalisations discursives concrètes, assurent des contextes aux valeurs envisagées. Par contre, les exemples de Saussure sont donnés en dehors de tout contexte discursif.

Certaines de ces différences contextuelles, notamment HOM., $\Rightarrow$, de et $\rightarrow$, sont indiquées dans l'organisation catégorielle de MERE puisqu'elles contribuent à la différenciation discursive. Ainsi, le contexte HOM., qui indique dans le Petit Robert les homonymes possibles, établit des rapports de contradiction entre les valeurs, leurs signifiés étant traditionnellement considérés comme distincts et ne se réalisant que l'un à l'exclusion de l'autre. Indiquant les renvois analogiques, autrement dit « un mot qui a un grand rapport de sens [...] avec le mot traité » (Tableau des termes, signes conventionnels et abréviations du dictionnaire. Le Petit Robert (version numérique), 2013), le contexte $\Rightarrow^{3}$ relie les termes qui le précèdent aux termes qui le suivent en affirmant le rapport d'implication de sens entre eux. À la différence de l'implication, où il est impossible de préciser l'orientation de la relation, la complémentarité permet de désigner le terme présupposant et le terme présupposé, comme il est possible de le faire dans les cas de dérivation et de composition (de) ou des termes apparentés $(\rightarrow$ dans les étymologies. Par conséquent, une typologie de ces différences contextuelles devient possible.

$\mathrm{Si}$, d'autre part, l'on tient compte de l'ordre dans lequel ces relations se constituent au sein du carré sémiotique, il devient possible d'envisager une considération hiérarchique entre HOM., $\Rightarrow$, de et $\rightarrow$ où le premier contexte indique l'infériorité de la valeur qu'il introduit, et les deux derniers la supériorité, tandis que $\Rightarrow$ marque une position supérieure par rapport au premier mais inférieure par rapport aux deux derniers. À leur tour, les contextes contribuent à l'articulation hiérarchique du sens.

C'est ainsi que la valeur postélaborée MERE intègre non une suite associative en dehors de tout contexte mais une association catégorielle élaborée en vue des contextes qui attribuent aux valeurs données de véritables rapports systémiques, notamment ceux d'homonymie, de renvoi analogique, de dérivation, de composition et de parenté étymologique.

$\mathrm{Si}$, toutefois, MERE est parmi les manifestations de l'archétype de la Mère, les autres réponses des mots croisés N17 ne le sont pas. Est-il alors possible de les considérer comme des valeurs génériques issues de la même division, notamment celle de la Mère ? Puisque la différenciation s'y fait à la base des qualités archétypales, il suffit alors de confirmer la présence de celles-ci au niveau des signifiés de toutes les réponses du N17.

Et c'est bien le cas car 21 qualités de l'archétype de la Mère, notamment alimentation, angoisse, autorité magique, bon, caché, croissance, dévore, élévation spirituelle au-delà de l'intellect, fécondité, impulsion secourable, inéluctable, monde des morts, obscur, patient, protecteur, renaissance, sagesse, secret, séduit, soutient, transformation magique (Jung, 1971:97), y compris les trois déjà mentionnées, sont présentées par des parties déterminées de leurs contenus sémantiques au niveau des signifiés de ces réponses :

Table 1

$$
\begin{aligned}
& \text { alimentation } \\
& \text { ALIMENTATION - ALIMENTER }-\Rightarrow \underline{\text { NOURRIR }}
\end{aligned}
$$

angoisse

ANGOISSE - CRAINTE

autorité magique 


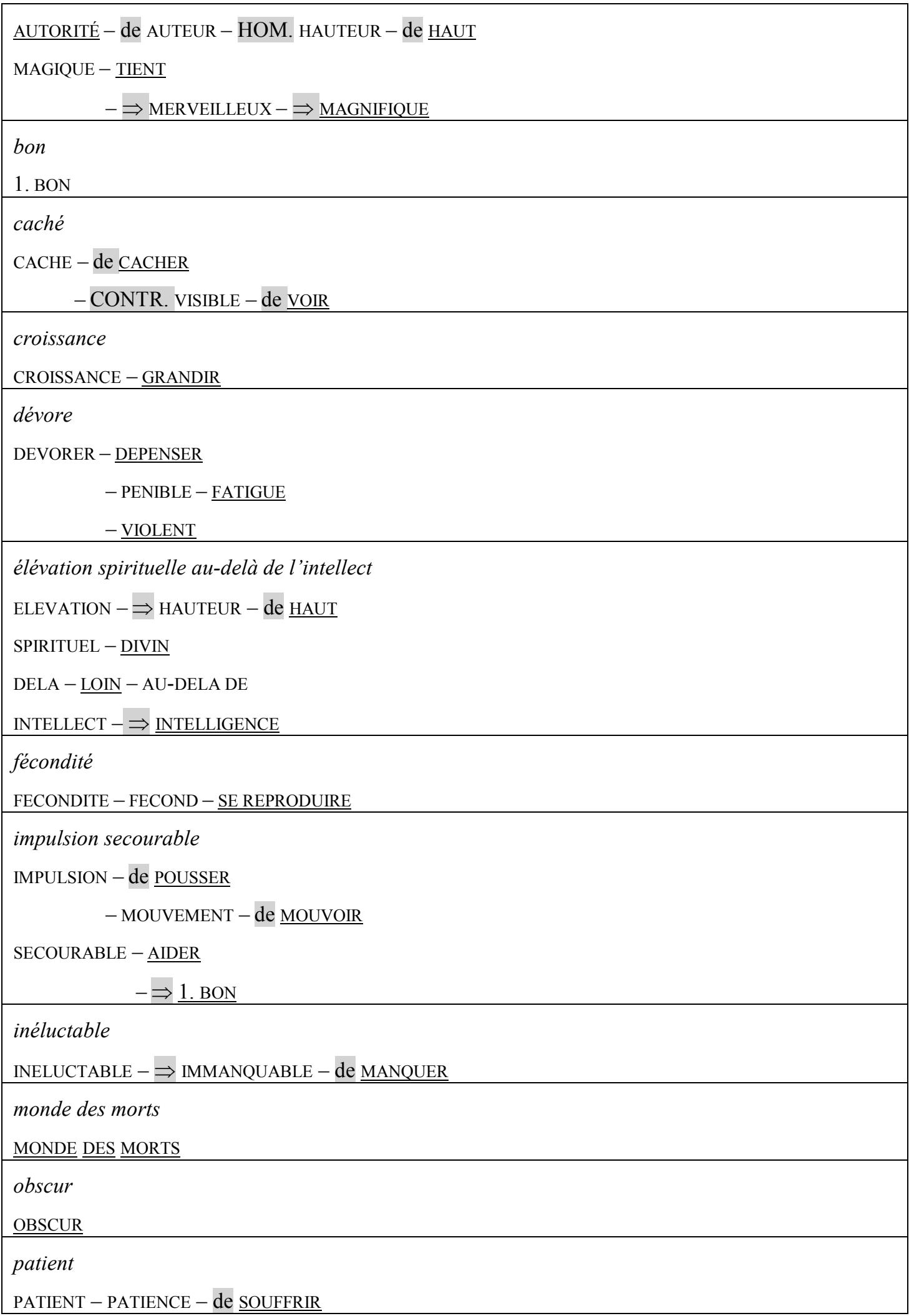




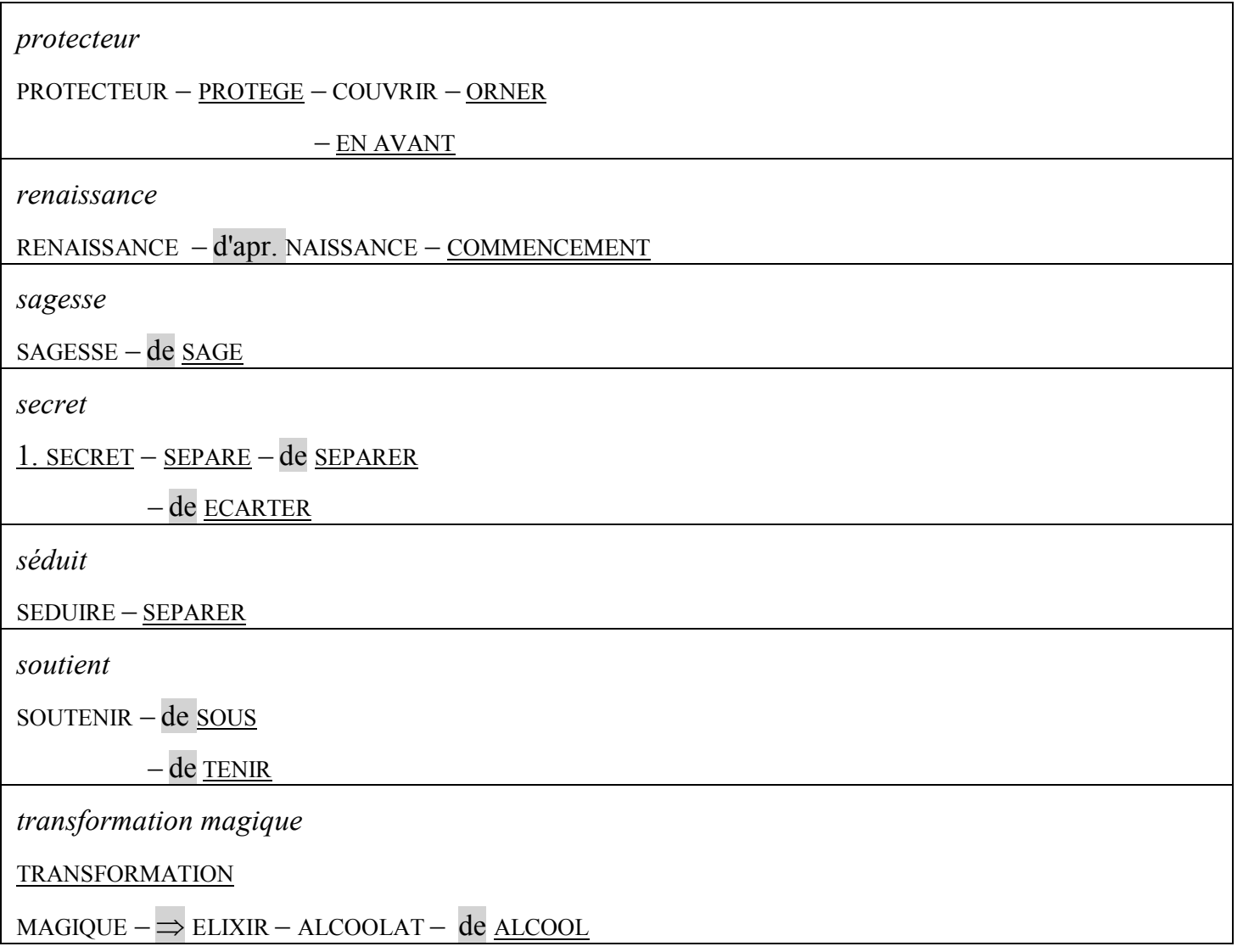

Se limitant à dix rangées horizontales (I - X) avec 16 réponses qui se croisent avec 16 autres disposées verticalement $(1-10)$, les mots croisés N17 de G. Perec fournissent au total 32 valeurs génériques qui poursuivent la division distinctive de la définition la Mère:

Table 2

CLASSEMENT - ORDONNANCE - DISPOSITION $-\rightarrow$ DISPOSER - DECIDER - TRANCHER - cf. ECARTER

HIVERNANTE - ne pas ETE - SAISON - du lat. SEMAILLES - de GRAINE - REPRODUCTION - SE REPRODUIRE

ABASOURDIR - FRAPPER -3 . AFFECTER $-\rightarrow$ AFFECT - ETAT - de TENIR - VIOLEMMENT - de VIOLENT

TETE DE MORT - YORICK... - 3. MORT - DEPOUILLE - VETEMENT - PROTEGER

-1. MORT $-\Rightarrow$ AU-DELA - $\underline{\text { MONDE }}$

ERAN - DERANGEMENT - CONTR. ORDRE - CONTR. CONFUSION $\rightarrow \rightarrow$ CONFUS - TUE - de PROTEGER

JURAI - SACRAI - $\Rightarrow$ BLASPHEMER - PROFERER - EN AVANT

LARES - GARDAIENT - PROTEGER

- CHAMBRE - PIECE - SEPAREE - de SEPARER

LM - SE SUIVENT - sans que MANQUE 


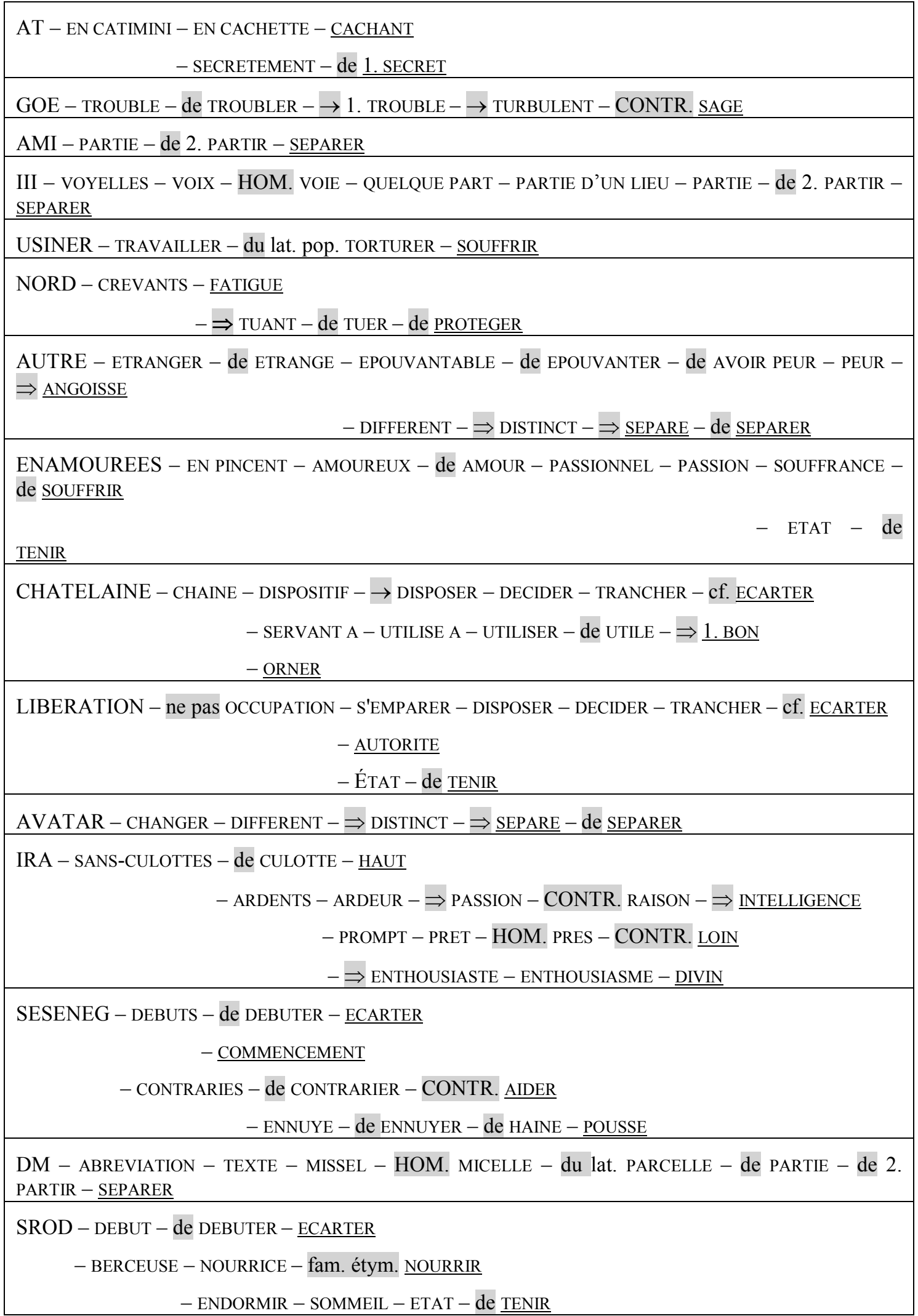




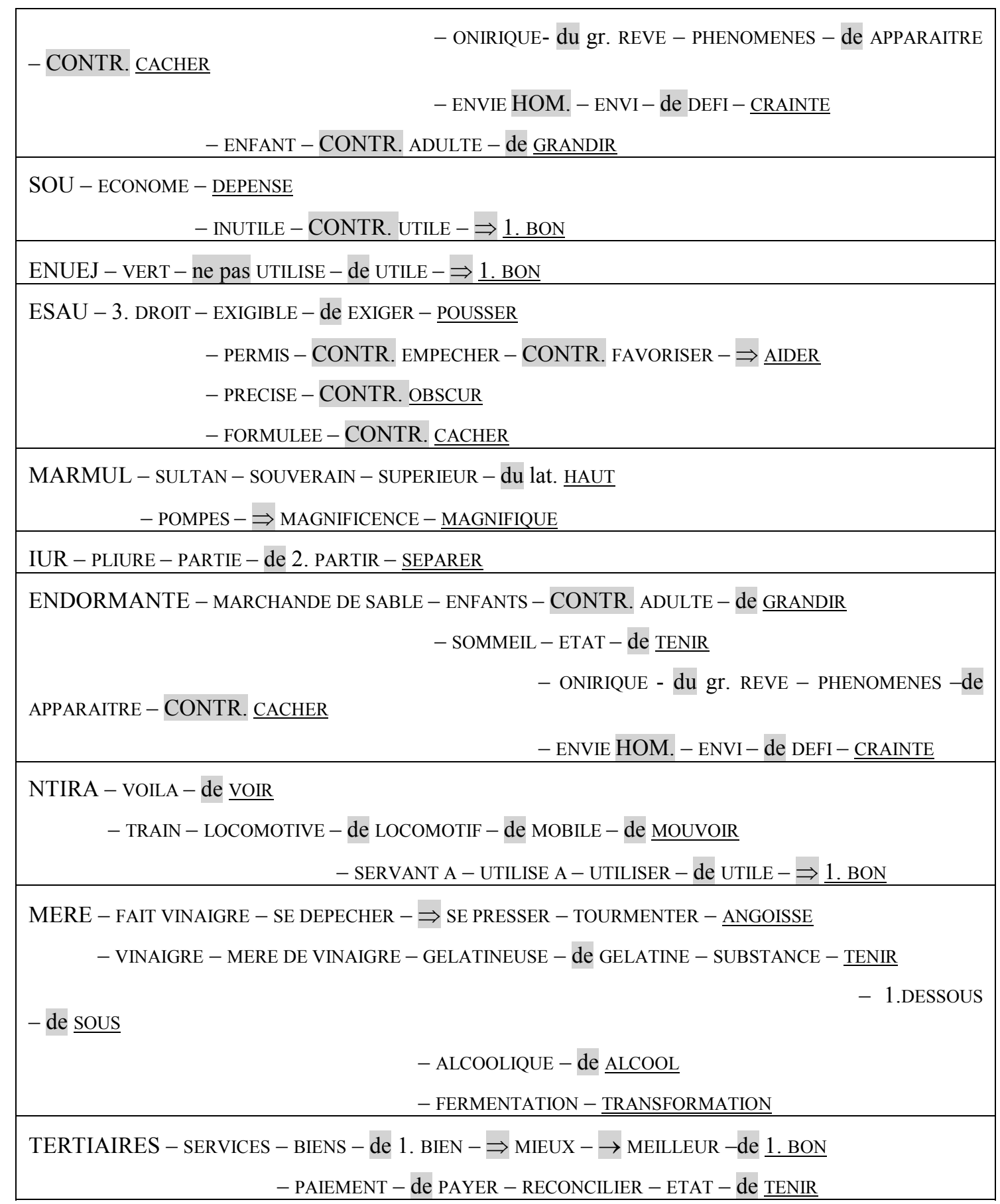

Quoique ne figurant pas parmi les manifestations de l'archétype de la Mère, les réponses du N17 s'avèrent telles puisque, fait mentionné à plusieurs reprises par Jung (1971), une énumération exhaustive des archétypes, de leurs manifestations et de leurs qualités est impossible. De même, le lexique d'une langue, et en particulier celui du français, ne représente pas non plus une liste exhaustive et fermée.

Souvent, plusieurs qualités archétypales participent à la différenciation d'une seule valeur générique pour y former plusieurs niveaux de différenciation et pour assurer l'articulation hiérarchique du sens. C'est notamment le cas d'ABASOURDIR, TETE DE MORT, LARES, AT, NORD, AUTRE, ENAMOUREES, 
CHATELAINE, LIBERATION, IRA, SESENEG, SROD, SOU, ESAU, MARMUL, ENDORMANTE, NTIRA, MERE, TERTIAIRES.

Cependant, même après la différenciation, des suites associatives de valeurs ainsi que de contextes paraissent « récursifs ». C'est le cas des associations :

DEBUT - de DEBUTER - ECARTER (dans le cas de SESENEG, SROD),

DISPOSER - DECIDER - TRANCHER - cf. ECARTER (CLASSEMENT, CHATELAINE, LIBERATION),

ENFANT - CONTR. ADULTE - de GRANDIR (SROD, ENDORMANTE),

TUER - PROTEGER (ERAN, NORD),

UTILE - $\Rightarrow \underline{1 . \text { BON }}($ CHATELAINE, ENUEJ, SOU, NTIRA)

ENVIE HOM. - ENVI - de DEFI - CRAINTE (SROD, ENDORMANTE).

Ces associations, à la suite de leur caractère commun, deviennent des généralisations. Les valeurs numériques y impliquées participent ainsi à la postélaboration de la Mère. De même, l'accident aristotélicien fait preuve de la définition. Toute généralisation ayant d'autre part un caractère abstrait, ces associations se chargent aussi d'une expression métalinguistique.

Ce sont toujours ces valeurs numériques communes à contenu sémantique partitif et marqué qui contribuent à la polysémie dans ETAT/ÉTAT - de TENIR (LIBERATION, TERTIAIRES, ABASOURDIR) où LIBERATION se réalise par ÉTAT - de TENIR à la différence de TERTIAIRES, ABASOURDIR qui se concrétisent par ETAT - de TENIR. De plus, l'on pourrait considérer ETAT - de TENIR comme faisant partie de SOMMEIL - ETAT - de TENIR (SROD et ENDORMANTE). Par conséquent, la polysémie s'organise sur l'axe de la postélaboration en acquérant de ce fait un caractère associatif et généralisateur. D'autre part, ETAT se différenciant d'ÉTAT, la récursivité totale est remise en cause. Et finalement, cette valeur numérique TENIR assure le sens polysémique de la définition cruciverbiste Ce n'est pas une occupation ! (LIBERATION).

La généralisation se fait parfois à la suite de plusieurs qualités. C'est ainsi que SEPARER est partagée entre

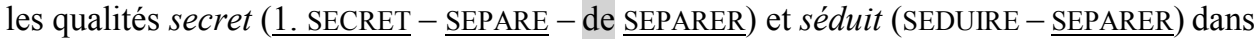

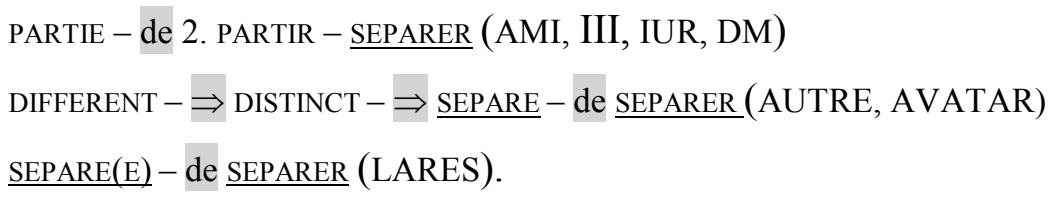

Ainsi, SEPARER devient valeur numérique commune et assure la généralisation qualitative : elle permet l'association de plusieurs valeurs génériques, autrement dit de plusieurs réponses cruciverbistes. Une généralisation devient alors possible dans les cas où il y a un partage de valeurs numériques qui sont des contenus sémantiques de différentes qualités archétypales.

Les contextes de ces associations communes, en particulier de, cf., CONTR., $\Rightarrow$, HOM., participent aussi à la généralisation. L'expression de la complémentarité et de l'implication par de et $\Rightarrow$ et de la contradiction par HOM. a déjà été mentionnée. L'expression de la contrariété par CONTR. s'impose par les termes contraires qu'il relie. Quant à cf., en indiquant « une expression, un terme de formation semblable, dans les étymologies » (Tableau des termes, signes conventionnels et abréviations du dictionnaire. Le Petit Robert (version numérique), 2013), celui-ci se charge d'associer des renvois étymologiques et d'exprimer ainsi une implication.

Il reste encore six contextes qui sont relevés dans le cas des valeurs du N17. Signifiant « dérivé de » dans une étymologie (Tableau des termes, signes conventionnels et abréviations du dictionnaire. Le Petit Robert (version numérique), 2013), du rappelle les rapports complémentaires instaurés par de. Des mêmes rapports se chargent aussi fam. étym., qui indique l'appartenance d'une valeur à une famille 
étymologique, ainsi que d'apr., qui signale l'influence formelle d'un terme sur un autre. Finalement, ne pas et sans que imposent la contradiction entre deux valeurs où l'une se présente toujours à l'exclusion de l'autre.

Si le nombre des contextes relevés se limite ici à 11 au total, il est bien possible que lors d'autres recherches de nouveaux contextes viennent s'ajouter, de sorte que se constitue une liste toujours ouverte et non exhaustive.

C'est aussi la considération hiérarchique de ces contextes qui contribue à la généralisation. Ainsi dans les cas des contextes de complémentarité et d'implication (CLASSEMENT, NORD, AUTRE, CHATELAINE, AVATAR, MARMUL, NTIRA, MERE, TERTIAIRES), la généralisation hiérarchique se fait au profit de la complémentarité, toute complémentarité étant une implication et non le contraire. En présence des contextes contradictoires et complémentaires (HIVERNANTE, III, DM), l'on aboutit à une complémentarité contradictoire, la contradiction précédant la génération de la complémentarité. Dans les cas des contextes d'implication et de contrariété (SOU), le contexte se définit sur l'axe des subcontraires, l'implication précédant la subcontrariété. Et toujours sur le même axe mais cette fois-ci en y ajoutant une détermination complémentaire, se définit la combinaison de contextes complémentaires et contraires (ERAN, GOE, SESENEG). Il arrive parfois que l'on trouve une combinaison de trois voire de quatre contextes différents. Dans le cas de LIBERATION et ENUEJ, la contradiction, l'implication, et la complémentarité se combinent selon les mêmes principes pour aboutir de nouveau à une complémentarité contradictoire. ENDORMANTE et SROD ont aussi des contextes de subcontrariété complémentaire à la suite de la combinaison des contextes contradictoires, complémentaires et contraires. Dans le cas d'ESAU, l'implication, la complémentarité et la contrariété se combinent pour aboutir aussi à une subcontrariété complémentaire. Enfin, en présence des quatre types de contextes à la fois (IRA), il est possible de considérer la première génération des termes catégoriels complétée.

La généralisation sur l'axe de la postélaboration se traduit ainsi par la hiérarchisation de la contradiction, l'implication, la complémentarité et la contrariété pour justifier la fonction métalinguistique de ces contextes dans le dictionnaire.

Ces contextes généralisés s'avèrent pourtant beaucoup plus «précis » si l'on tient compte de leur ordre successif et des valeurs exprimées. Il suffit de revoir le cas des contextes combinant la complémentarité et l'implication (CLASSEMENT, NORD, AUTRE, CHATELAINE, AVATAR, MARMUL, NTIRA, MERE, TERTIAIRES) pour constater que d'une part, il existe des valeurs numériques (ORNER dans CHATELAINE, FATIGUE dans NORD, TRANSFORMATION dans MERE) qui ne sont introduites par aucun de ces deux contextes, et que d'autre part, il y a des valeurs numériques qui ne sont introduites que par des contextes d'implication (MAGNIFIQUE dans MARMUL, ANGOISSE dans MERE) ou de complémentarité (HAUT dans MARMUL, VOIR et MOUVOIR dans NTIRA, TENIR, SOUS et ALCOOL dans MERE, TENIR dans TERTIAIRES). Les signifiés ainsi postélaborés des 32 valeurs génériques vont se voir finalement marqués par différentes valeurs numériques qui entretiennent de véritables relations systémiques de négation, homonymie, exclusion, renvoi étymologique, synonymique et contraire, dérivation et parenté pour faire preuve d'une diversité sémantique sur l'axe de la différenciation discursive en excluant tout récursivité totale.

Si l'axe de la différenciation se fait par la marque qualitative, l'axe de la postélaboration se constitue par le rapport partie-tout: des valeurs numériques et des contextes s'intègrent dans les signifiés des valeurs hyperonymiques. Alors, il s'agit d'une postélaboration des signifiés des réponses cruciverbistes à partir de leurs définitions. Des associations récursives, constituées à la base de valeurs numériques, assurent d'autre part la généralisation sur cet axe et relient des valeurs génériques. Alors, il est question d'associations entre les réponses d'un discours cruciverbiste.

La diversité discursive assurée par les valeurs ainsi postélaborées se traduit dans des procédés d'élaboration des définitions cruciverbistes. Les deux premiers de ces procédés décrits par Perec (2012 [1999]:19-24) consistent dans l'établissement d'une équivalence entre les réponses et leurs définitions, et sont des procédés de premier degré. L'un consiste à utiliser les définitions fournies par des dictionnaires, le plus souvent en les abrégeant, et d'en extraire les réponses. Tel paraît être le cas de CLASSEMENT, de 
CHATELAINE et, dans une certaine mesure, de LARES et TERTIAIRES, les définitions des deux dernières solutions étant une sorte d'équivalents paraphrastiques des définitions du Petit Robert. Il faut y ajouter USINER, dont la définition est élaborée à partir de son étymologie, et ESAU où l'auteur semble avoir raccourci la définition de type biblique fournie à titre d'exemple par le Grand Robert (cf. aînesse). De pareils exemples de raccourcis paraphrastiques mais de sens opposé sont donnés par les définitions d'HIVERNANTE et de LIBERATION, tandis que celles de JURAI et AUTRE représentent des renvois synonymiques. Le recours à un procédé de synonymie a aussi lieu dans le cas d'ENAMOUREES mais moyennant amoureux, c'est-à-dire, à l'aide d'un terme intermédiaire dans l'équivalence aristotélicienne entre des univoques. Tel est aussi le cas de SOU et d'une partie de sa définition (l'économe) : le terme intermédiaire est cette fois-ci un proverbe (Un sou est un sou) d'où, de surcroît, la réponse est extraite.

Retrouver la réponse MARMUL à la suite de sa définition implique plutôt des connaissances géographiques de même que TETE DE MORT demande des connaissances sur les personnages d'Hamlet, ou du moins sur celui de Yorick.

C'est toutefois le deuxième procédé de premier degré qui est illustré par Perec à travers des exemples de proverbes. Pour le dire autrement, il s'agit de prendre des expressions à caractère figé pour élaborer des définitions : ainsi la locution familière marchande de sable pour ENDORMANTE.

S'il est plus ou moins possible de trouver l'équivalence entre les réponses et les définitions de premier degré attestée par les dictionnaires, avec marchande de sable (ENDORMANTE) l'on empiète sur les définitions de degrés supérieures, qui reposent sur l'ambivalence, pour déborder ainsi sur la singularité du cruciverbiste : un renvoi synonymique entre marchande de sable et ENDORMANTE relève plutôt de l'usage singulier, et il est donc difficile qu'il soit attesté par un dictionnaire.

L'un des procédés employé à partir du deuxième degré consiste alors à « désigner le mot à découvrir (le signifié) par un signifiant qui désigne habituellement autre chose » (Perec, 2012 [1999]:20). Il semble par conséquent que le cruciverbiste non seulement brise le lien attesté entre le signifié et son signifiant mais renverse aussi leurs statuts: le lecteur doit considérer la réponse NORD comme le signifié de son signifiant-définition Ses pavés sont crevants alors que les dictionnaires procèdent plutôt dans le sens inverse. Il est alors possible d'entrevoir certains procédés connotatifs (Barthes, 1993:1517-1519) où crevant représente à son tour un signifiant et un signifié polysémique, et c'est cette polysémie qui renvoie, d'une part, par mourir au signifiant ENFER, dont le signifié atteste le proverbe L'enfer est pavé de bonnes intentions, et d'autre part, par l'expression familière crever de froid au nouveau signifié NORD. Il ne faut pas oublier pour autant que c'est la même valeur crevants qui s'associe aux deux valeurs numériques FATIGUE et PROTEGER. De même, la définition d'AVATAR se fait par une synonymie inhabituelle à la suite de l'expression Ça vous change un homme : il est toutefois possible d'en trouver une certaine attestation dans les dictionnaires pourvu que l'on retienne avatar dans son contresens de mésaventure.

Dans un désir d'éviter l'approche sémantique d'un nom propre qu'est la définition Yorick, le même principe du renversement du rapport signifié - signifiant est ici adopté : l'on est parti du signifié-réponse TETE DE MORT pour poursuivre la division.

La singularité du cruciverbiste aboutit même à l'établissement de ses propres polysémies. Par un autre procédé de deuxième degré qui consiste à désigner «le signifié en mélangeant dans la même définition deux ou trois signifiants habituellement séparés » (Perec, 2012 [1999]:20), IRA se voit responsable d'exprimer le sentiment de colère (lat. ira) des sans-culottes et des tenants d'IRA (Armée républicaine irlandaise) à la fois. De même que MERE peut bien se dépêcher en faisant vinaigre (expression fam.) et en se constituant en membrane gélatineuse à la surface d'un liquide alcoolique.

Perec crée aussi ses métadéfinitions ou des «définitions trouvant leurs références dans le vocabulaire même des mots croisés » (Perec, 2012 [1999]:21) : la réponse VIII (horizontalement) n'est en effet que la suite de trois voyelles III. Il ne faut pas sous-estimer d'autre part les chevilles que sont AT, ERAN, DM, LM, IUR et auxquelles l'on peut ajouter le cas d'ABASOURDIR : il n'y manque pas non plus une cheville qui permet de faire rimer fort de la définition cruciverbiste avec mort de l'expression frappé à 
mort pour établir ainsi des rapports synonymiques, moyennant un terme intermédiaire qu'est tuer entre ABASOURDIR et frapper.

L'auteur invente aussi ses anagrammes de type NTIRA et AMI : forgées à la suite de train et de familles, ces réponses sont extraites de leurs définitions Voilà ce qui arrive quand on rate le train! et Fait effectivement partie de la famille dont elles font vraiment partie.

Il est plutôt difficile d'attribuer un caractère anagrammatique à GOE, SESENEG, SROD, ENUEJ en observant leurs définitions cruciverbistes. Ces réponses rappellent dans une certaine mesure une sorte de verlan singulier. Dans de pareils cas, le cruciverbiste se contente simplement «d'en donner la définition très exacte » (Perec, 2012 [1999]:24). En effet, ego a vraiment un peu troublé l'ordre de ses lettres (cf. Un mot un peu troublé dans son latin) et genèses se sont constituées dans le sens contraire (cf. Des débuts bien contrariés) tout en entretenant des relations synonymiques avec débuts moyennant la suite genèse naissance - commencement - début: genèse signifie étymologiquement naissance dont II. Fig. 1. contient commencement qui renvoie à début (Le Petit Robert (version numérique), 2013). C'est au même titre que sont à envisager les envers de dors et jeune: SROD (cf. Dans un sens, c'est un début de berceuse) et ENUEJ (cf. Vert à l'envers). Dans le premier cas, la suite synonymique est berceuse endormir - dormir. Dans le second, le renvoi à vert est plutôt attesté par les dictionnaires, sans oublier cependant les rimes.

Ces valeurs postélaborées lors de l'établissement de l'équivalence entre les définitions et leurs réponses contribuent au fonctionnement des opposés, des renvois synonymiques, des équivalences paraphrastiques, de même qu'à l'usage familier et à la constitution des locutions, des paraphrases définitionnelles et des définitions étymologiques, en permettant aussi un certain contenu encyclopédique à caractère biblique, géographique et littéraire qui empiète parfois sur le domaine des noms propres.

Et il devient possible de trouver la réponse MERE dans un dictionnaire où des valeurs numériques, entre lesquelles ANGOISSE, TENIR, SOUS, TRANSFORMATION et ALCOOL, sont des mots-clefs, et où des valeurs spécifiques renvoient vers ces mots-clefs. Chaque renvoi peut conduire vers un mot-clef puisqu'il le contient implicitement dans son signifié : ainsi FAIT VINAIGRE, SE DEPECHER, SE PRESSER, PRESSER, TOURMENTER et SE TOURMENTER vont renvoyer à ANGOISSE, ALCOOLIQUE à ALCOOL et 1 . DESSOUS à SOUS, tandis que GELATINEUSE, GELATINE et SUBSTANCE vont mener à TENIR et à SOUS à la fois pour respecter ainsi l'articulation hiérarchique du sens. Toujours en respect de cette hiérarchie, vINAIGRE et MERE DE VINAIGRE vont conduire à ALCOOL, TENIR et SOUS à la fois. Si l'on y applique l'existence de plusieurs contextes, ces renvois vont se faire non seulement en termes de synonymie, mais aussi, par ex. en termes de dérivation (1. DESSOUS - de SOUS, ALCOOLIQUE - de ALCOOL). Dans les cas où une valeur numérique relève des signifiés de plusieurs qualités archétypales, par ex. SEPARER, des définitions simples, notamment secret et séduit, accompagnant ce mot-clef, peuvent affiner la recherche de la réponse. En effet, les associations entre les réponses et leurs définitions et les associations entre les réponses elles-mêmes peuvent s'organiser en un « dictionnaire à l'envers » (Greimas, 1970:287).

Et il importe surtout de ne pas oublier que lors de ces élaborations des réponses et leurs définitions, des valeurs archétypales, à partir des génériques jusqu'aux numériques, sont mises en jeu de sorte que l'élaboration singulière est encadrée par la postélaboration. Et bien à l'envers : celle-ci se fait lors de la constitution singulière des mots croisés N17. Ce qui permet bien aux lecteurs d'en trouver les réponses. Et ce qui permet à Perec de constituer son propre vocabulaire cruciverbiste. La preuve d'un tel lien entre l'élaboration singulière et la postélaboration, entre l'auteur et ses lecteurs, est un argument en faveur de la nature psychique et collective des valeurs archétypales.

Il est alors possible que le sens se constitue en termes de valeurs dans le cadre d'une sémantique des valeurs archétypales, où celles-ci sont issues d'une même division pour devenir ainsi des valeurs génériques, spécifiques et numériques.

Lors de cette division, des niveaux de différenciation s'établissent lorsqu'une nouvelle valeur numérique intervient : l'articulation hiérarchique du sens est ainsi signalée. Un axe de la différenciation se définit alors. Cet axe se distingue par la présence des valeurs numériques. Celles-ci étant des parties marquées 
des signifiés des qualités archétypales, c'est la différence qualitative qui assure l'articulation du sens. De différents contextes contribuent aussi à cette articulation. Cet axe assure ainsi la diversité discursive.

La différenciation faite, des processus de postélaboration interviennent. C'est d'une part la postélaboration des signifiés: elle consiste à intégrer dans les valeurs les différences systémiques établies. L'intégration des valeurs numériques communes assure alors le fonctionnement du principe partie-tout sur cet axe. Cette intégration assure d'autre part la récursivité d'une partie des associations pour permettre ainsi la généralisation et la hiérarchisation. Et celles-ci rendent possible la postélaboration des catégories sur cet axe.

$\mathrm{Si}$ le parcours associatifs est assuré par des valeurs numériques issues des signifiés des qualités archétypales, la nature psychique et collective des autres valeurs, spécifiques et génériques, se voit justifiée. Par conséquent, la notion de valeur devient opératoire au niveau sémantique, une valeur étant générique, spécifique ou numérique par son occurrence, et archétypale par sa nature.

\section{Références bibliographiques:}

Aristote. (1967). Topiques. 1. Livres I-IV (texte établi et traduit par Jacques Brunschwig). Paris : Les Belles Lettres.

Aristote. (2007). Topiques. II. Livres V-VIII (texte établi et traduit par Jacques Brunschwig). Paris : Les Belles Lettres.

Attali, Alain. (2008). Quand le Sphinx fait de l'humour: Georges Perec verbicruciste. Enseigner le français, N8, pp.67-71.

Barthes, Roland. (1993). Eléments de sémiologie. Euvres complètes, tome I, 1942-1965 (éd. établie et présentée par Éric Marty). Éditions du Seuil, pp. 1465-1524.

Greimas, Algirdas Julien \& Courtés, Joseph. (1979). Sémiotique. Dictionnaire raisonné de la théorie du langage. Paris : Hachette.

Greimas, Algirdas Julien. (1970). L'écriture cruciverbiste. Du sens, essais sémiotiques. Paris : Éditions du Seuil, pp. 285-308.

Jakobson, Roman. (1963). Les embrayeurs, les catégories verbales et le verbe russe. Essais de linguistique générale. Les fondations $d u$ langage (trad.de l'anglais et préfacé par Nicolas Ruwet). Paris : Les Éditions de Minuit, pp.176-196.

Jakobson, Roman. (1971). Parts and Wholes in Language. Selected Writings II: Word and Language. The Hague : Mouton \& Co. Publishers, pp.280-284.

Jeandillou, Jean-François. (1995). Verbigérations cruciverbistes : pour un dialogisme énigmatique dans les mots croisés de Georges Perec. Le Cabinet d'amateur. Revue d'études perecquiennes, N4, pp. 75-96.

Jung, Carl Gustav. (1971). Les racines de la conscience. Études sur l'archétype. (trad. de l'allemand par Yves Le Lay. Ouvrage publié sous la direction du Dr Roland Cahen). Paris : Éditions Buchet / Chastel.

Kleiber, Georges. (1990). La sémantique du prototype. Paris : PUF.

Kleiber, Georges. (1994). Nominales : essais de sémantique référentielle. Paris : Armand Colin.

La Ferté, Roger \& Capelovici, Jacques. (1975). Pratique des mots croisés. Paris : PUF.

Örsi, Tibor. (1996). Analyse lexicologique des mots croisés français. Revue d'études françaises, vol.1, pp.93-99.

Perec, Georges. (2012 [1999]). Les mots croisés : précédés de considérations de l'auteur sur l'art et la manière de croiser les mots. Paris : P.O.L, Gallimard.

Saussure, Ferdinand de. (1955 [1916]). Cours de linguistique générale. Paris : Payot.

Saussure, Ferdinand de. (2002). Écrits de linguistique générale. Gallimard.

Wittwer, Jacques. (2004). Mots croisés et psychologie du langage. Paris, Budapest, Torino : l'Harmattan. 


\section{Dictionnaires:}

Dictionnaire des mots croisés et fléchés. (2011). Paris : Larousse.

Dictionnaire des mots croisés, mots fléchés \& autres jeux de lettres. (2006). Paris : Dictionnaires Le Robert.

Le Grand Robert de la langue française (version électronique). (2005). Paris : Dictionnaires Le Robert.

Le Petit Robert des noms propres. (2011). Paris : le Robert.

Le Petit Robert, dictionnaire alphabétique et analogique de la langue française (version numérique). (2013). Paris : Dictionnaires Le Robert.

${ }^{1}$ Le signe * est introduit ici pour distinguer la complémentarité de l'implication.

${ }^{2}$ Notre choix des mots croisés N17 est motivé par la présence de la réponse MÈRE. Ainsi, nous nous distinguons des allusions possibles avec l'intervention du nombre 17 dans l'œuvre de G. Perec (v. Mazzorato, Wilfrid \& Raysséguier, Serge. (1995). L'intervention du dix-sept. Le Cabinet d'amateur. Revue d'études perecquiennes, N4, pp.97-108.).

${ }^{3}$ Nous remplaçons par ce signe la flèche qui introduit les renvois analogiques dans Le Petit Robert (version numérique) (2013). Cette flèche ressemble en effet beaucoup à celle dans les étymologies. 